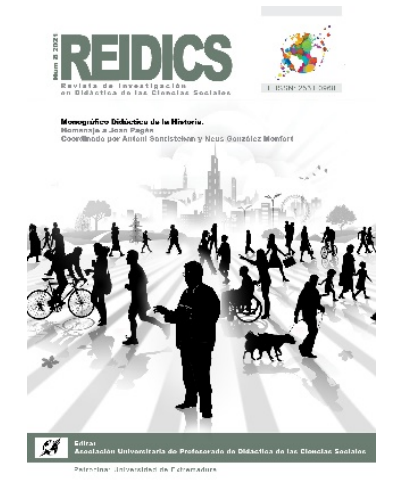

\title{
REIDICS
}

Revista de Investigación en

Didáctica de las Ciencias

Sociales
Núm. 8, 2021

Recibido 30 septiembre de 2020

Aceptado 03 enero 2021

E-ISSN: 2531-0968

\section{Aprendizaje basado en objetos en Educación Infantil: evaluación de una estrategia de intervención}

\author{
Object based learning in Early Years Education: evaluative study
}

of a teaching intervention

\author{
Mónica Cava Pagán \\ Universidad de Murcia \\ Email: monicacava1992@gmail.com \\ ORCID: https://orcid.org/0000-0001-7612-4438 \\ Laura Arias Ferrer \\ Universidad de Murcia \\ Email:larias@um.es \\ ORCID: https://orcid.org/0000-0003-3121-1882
}

DOI: https://doi.org/10.17398/2531-0968.08.224

\section{Resumen}

Las fuentes objetuales pueden ser un recurso idóneo para trabajar contenidos históricos en edades tempranas: se trata de elementos tangibles que permiten la experimentación directa e introducen tiempos ajenos a la realidad del estudiante de una manera motivadora. Sin embargo, son escasas las investigaciones evaluativas realizadas al respecto, y es ahí donde reside la importancia de esta investigación. Esta se centra en el análisis y evaluación de los resultados de aprendizaje obtenidos tras la implementación de una intervención una intervención didáctica diseñada ad hoc. Se pretenden concretar así las implicaciones educativas que la didáctica del objeto posee para el desarrollo de las habilidades de pensamiento. Para ello, se ha realizado una investigación cuasi experimental de corte cualitativo. Los datos han sido recopilados mediante observación participante y el análisis de las intervenciones de los participantes se ha realizado a través de su registro audiovisual. La motivación del alumnado se midió mediante una escala de valoración. La intervención se realizó con un total de 19 alumnos y alumnas de cinco años, todos ellos adscritos a un mismo grupo-clase, de un centro de adscripción pública de Educación Infantil. Tras la implementación, se ha podido apreciar una mejora por parte de los estudiantes participantes de las habilidades de observación así como una interacción más completa y compleja con las fuentes. Esto lleva a pensar que la realización de este tipo de propuestas en etapas infantiles favorece en el alumnado el desarrollo de habilidades como las descritas. 
Palabras clave: educación formal; educación de la primera infancia; enseñanza de la historia; patrimonio cultural.

\begin{abstract}
Objects can be an ideal resource to work with historical contents at an early age since they are tangible elements that let students experience directly and allow the introduction of the past in a motivating way. However, evaluative research regarding the analysis of the benefits that such strategy has are scarce. Here lies the importance of this research. This research focuses its attention on the analysis and assessment of the learning outcomes achieved by the students after the implementation of a teaching intervention designed ad hoc for this study. The final aim of this study is to specify the educational implications that object based learning strategies has for the development of thinking skills. This is a quasi-experimental study with a qualitative approach. The data have been collected through participant observation, participant intervention recordings, and a rating scale developed by the students. The intervention was carried out in a 5-year-old classroom, with a total of 19 participants. After the implementation, an improvement can be seen in relation to certain observation and description skills and the process of interaction with the sources. The students also expressed the high level of motivation they experienced. All this leads to think that the introduction of these strategies in Early Years Education favor the development of the mentioned thinking skills among the young learners.
\end{abstract}

Keywords: formal education; early childhood education; history education; cultural heritage.

\title{
1. Introducción
}

Es cada vez más habitual encontrar en las aulas de Educación Infantil contenidos de índole histórica debido principalmente al carácter transversal e interdisciplinar de sus temáticas, lo que facilita la integración de diferentes áreas de conocimiento y el desarrollo de metodologías como el aprendizaje basado en proyectos (Miralles y Rivero, 2012). Sin embargo, es usual apreciar en esta etapa que el contacto existente entre el alumnado y la historia se reduce únicamente a la realización de estos proyectos que, en general, no poseen un vínculo directo con los alumnos (Casanova, Arias y Egea, 2018).

La investigación que a continuación se desarrolla tiene su justificación en la necesidad de valorar los beneficios que la introducción de la historia en las aulas de Educación Infantil de una manera activa puede poseer. Para ello, el trabajo con fuentes objetuales se muestra como un recurso idóneo: los objetos son elementos tangibles que permiten la experimentación directa y que además introducen en el aula tiempos ajenos a la realidad del estudiante de una manera motivadora. La investigación atribuye a la didáctica del objeto múltiples beneficios al permitir construir un aprendizaje activo, creativo y significativo desde edades tempranas, asociados al desarrollo de una estrategia interrogativa donde el alumnado puede establecer sus propias observaciones, deducciones, hipótesis y conclusiones, todo ello a través de un proceso de investigación que le permitirá construir su propio conocimiento (Cooper, 2002; Santacana y Llonch, 2012).

Sin embargo, son escasas las investigaciones evaluativas realizadas al respecto, y es ahí donde reside la importancia de esta investigación. En concreto, esta investigación pretende 
evaluar los aprendizajes obtenidos tras el desarrollo en un aula de cinco años de una experiencia basada en la didáctica del objeto y diseñada a partir de la realidad histórica de su localidad. Para ello, se analiza el desarrollo de la habilidad del pensamiento histórico asociada a la introducción y uso de fuentes y pruebas históricas a través de la observación, interacción y establecimiento de hipótesis realizadas por los estudiantes participantes fruto de su interacción con fuentes arqueológicas. Esta investigación se inserta en el marco del proyecto Re-construyendo la historia (Egea, Pernas y Arias, 2014). Asociada a esta línea de trabajo, se han llevado a cabo diversas investigaciones basadas en el análisis de intervenciones didácticas en aulas de Educación Infantil para trabajar el pensamiento histórico a través del uso de fuentes o pruebas (Arias y Casanova, 2018a; Arias et al., 2016). Esta investigación, sin embargo, amplía las anteriores al analizar el proceso concreto de indagación que el estudiante realiza en el momento que afronta el ejercicio analítico que se le propone. La investigación se centra por lo tanto en comprobar si el alumnado de Educación Infantil desarrolla habilidades de observación y análisis (a través de la interacción con la fuente) así como si se aprecia una evolución en la génesis de inferencias.

\section{Marco teórico}

¿Es posible trabajar las denominadas habilidades del pensamiento histórico con estudiantes de Educación Infantil? ¿Qué tipo de estrategia es la más adecuada para empezar a sentar las bases del futuro estudiante de historia? Estamos convencidos que para pensar la historia se necesitan crear rutinas ya en edades tempranas. Sin embargo, es fundamental introducir de manera paulatina cada uno de los ejercicios analíticos que se requieren y para ello la selección de recursos se muestra fundamental (Cooper, 2002).

Al respecto, Bruner $(1960,1999)$ anima al diseño e introducción de experiencias que supongan desafíos para los estudiantes para promover así su desarrollo intelectual independientemente de la edad del estudiante. Pero, para ello, es necesario contar con ciertas premisas que garanticen la idoneidad de la estrategia, actividad o recurso utilizada. Al respecto, se muestra fundamental la motivación e implicación del alumnado en el aprendizaje, la creación de un adecuado clima de interacción, la posibilidad de recrear contextos relevantes y significativos para el alumnado, y ser conscientes de que el pensamiento no es un elemento estático ya que puede ser desarrollado por el alumnado en múltiples vertientes (Wallace, 2002). Solo así conseguiremos que el alumnado se apropie de la tarea a realizar y que se involucre en el proceso.

Ejercicios sencillos, pero desafiantes, a través de los cuales el alumnado pueda expresar opiniones, tomar decisiones, argumentar el porqué de sus acciones, esbozar inferencias, realizar deducciones, etc. a partir de temáticas cercanas a la realidad del alumnado fomentan el incipiente desarrollo de las habilidades del pensamiento. En este sentido, Maine (2015) destaca los beneficios de realizar esta tarea en conjunto favoreciendo el aprendizaje dialógico y cooperativo. Se ha de establecer pues la posibilidad de que los alumnos piensen en alto, que contrasten sus respuestas con las apreciaciones de sus compañeros, que sea posible repensar y revaluar sus conocimientos (Pappas, Kiefer y Levstik, 2006), así como compartir experiencias y construir significados de manera cooperativa. 
Por todo ello, no podemos más que sino parafrasear el título con el que Wallace (2002) inicia su reflexión sobre el desarrollo de las habilidades de pensamiento en edades tempranas, "The earlier we start the better!". De hecho, cada vez son más los autores que destacan la posibilidad de enseñar historia en edades tempranas (Cooper, 2002; De Groot-Reuvekamp, Boxtel, Ros y Harnett, 2014).

A favor de esta introducción temprana de la disciplina, diversos autores suelen esgrimir que los niños en edad infantil están conectados a su propio pasado a través de los familiares, del contexto que los rodea, de los medios de comunicación y de la cultura de su entorno, además de que estos poseen una curiosidad natural sobre su pasado (Dean, 2008; Wood y Holden, 2007). Cómo realizar un aprendizaje significativo de la misma se vuelve más complejo. Aunque son diversas las publicaciones que recogen múltiples propuestas en relación a cómo introducir la historia en las aulas de infantil (Cooper, 2013; Wood y Holden, 2007), son todavía escasas las investigaciones que determinen los beneficios específicos que el desarrollo de dichas estrategias poseen en estudiantes de Educación Infantil o primeros años de Primaria (Arias, Egea y Levstik, 2019; De Groot-Reuvekamp et al., 2014; Harnett, 1993; Lee y Ashby, 2000; Levstik y Barton, 2015; Solé, 2009; VanSledright y Brophy, 1992).

En Educación Infantil esto se puede conseguir a través de diferentes elementos como son la didáctica del objeto, las imágenes y el patrimonio (Prats y Santacana, 2011), entre otros. Esta investigación se centra en concreto en la introducción del objeto como fuente de información sobre el pasado. Las fuentes materiales, los objetos, poseen determinadas características que las hacen atractivas y útiles en el contexto escolar: son elementos tangibles que facilitan la experimentación directa, son un fuerte elemento motivador para los discentes, permite la introducción de temáticas y conceptos variados y favorece el desarrollo de la imaginación y el pensamiento creativo, entre otras cosas (Arias et al., 2016; Cooper, 2002; Santacana y Llonch, 2012; Seixas y Morton, 2013; Wood y Holden, 2007). Se destaca igualmente el hecho de que son de las pocas fuentes primarias que pueden ser introducidas en esta etapa precisamente por su carácter no textual (Ashby, 2011).

Pero no se debe olvidar que el éxito de esta estrategia está ligado al uso reflexivo, interrogativo, creativo y abierto de este recurso, ya que por sí solas no ofrecen información y pueden caer en un uso asociado meramente al entretenimiento o como elemento decorativo o ilustrativo de los contenidos narrados. Es por ello que, para un correcto uso de las fuentes en el aula, hay que iniciar a los niños en el método de investigación que favorezca la interrogación del objeto, el pensamiento crítico, el debate y el diálogo en grupo (Cooper, 2002; Santacana y Llonch, 2012; Seixas y Morton, 2013). Es fundamental partir de las preguntas adecuadas que nos permitan interrogar las fuentes y analizar cuidadosamente cada uno de sus aspectos. Acostumbrar al alumnado a realizar estas preguntas les va a animar, sin duda, a preguntar, a observar o a pensar históricamente (Levesque, 2008; Seixas y Morton, 2013).

Podemos encontrar diferentes aproximaciones al análisis de los objetos dependiendo del autor de consulta (Cooper, 2002; Santacana y Llonch, 2012; Seixas y Morton, 2013). Sin embargo, todos comparten una similar estructura que ha sido recogida por Egea, Pernas y Arias (2014) y que resumimos en la tabla 1 . De manera general, para analizar el objeto hay que descomponerlo 
en todas sus partes (Santacana y Llonch, 2012), lo que nos permite conocer cómo es un objeto, cómo se comporta, cuál es su función, cómo se ha fabricado, etc. Por lo tanto, este análisis obliga a los alumnos a fijarse en los detalles y poder relacionarlo después con otros conceptos más generales.

Tabla 1

Análisis de fuentes objetuales: fases, acciones y preguntas

\begin{tabular}{|c|c|c|}
\hline Fase & Acciones & Preguntas \\
\hline $\begin{array}{l}\text { Observación, } \\
\text { manipulación y análisis } \\
\text { de los aspectos } \\
\text { formales }\end{array}$ & $\begin{array}{l}\text { Se debe observar y manipular el objeto para } \\
\text { analizar su estructura y morfología: color, } \\
\text { forma, material, decoración, etc. }\end{array}$ & $\begin{array}{l}\text { ¿Cómo es?, ¿qué forma tiene? } \\
\text { ¿de qué material está hecho?, } \\
\text { ¿está decorado o tiene } \\
\text { dibujos?, ¿de qué color es? }\end{array}$ \\
\hline $\begin{array}{l}\text { Planteamiento } \\
\text { hipótesis }\end{array}$ & $\begin{array}{l}\text { Se debe realizar deducciones e inferencias a } \\
\text { partir de las fuentes analizadas, en lo } \\
\text { referido a su elaboración (análisis técnico), } \\
\text { función (análisis funcional), contexto y su } \\
\text { significado o valor }\end{array}$ & $\begin{array}{l}\text { ¿Quién y cómo fue elaborado?, } \\
\text { ¿qué es y para qué sirvió?, ¿de } \\
\text { quién era?, ¿piensas que es un } \\
\text { objeto importante? }\end{array}$ \\
\hline
\end{tabular}

Fuente: elaboración propia a partir de Egea, Pernas y Arias (2014).

En estas premisas se basa la intervención analizada en esta investigación, diseñada ad hoc para la misma, cuyos resultados nos permite ser optimistas en relación con las múltiples posibilidades que esta estrategia en particular, y la introducción de contenidos históricos en general, posee para romper con la visión tradicional de la enseñanza de la historia y el desarrollo de las habilidades de pensamiento.

\section{Marco empírico}

El objetivo principal de esta investigación fue analizar y evaluar las implicaciones educativas del uso de fuentes primarias en un aula de Educación Infantil, a partir de una intervención basada en la didáctica del objeto. Para poder responder al objetivo general planteado se mostró necesario (1) analizar la capacidad de observación del alumnado a través de preguntas sobre los objetos, (2) conocer el grado de interacción que los alumnos establecen a la hora de manipular, observar y analizar las fuentes objetuales, (3) valorar la capacidad de utilizar fuentes objetuales para realizar hipótesis sencillas y establecer algunas conclusiones en torno a las mismas y, por último, (4) valorar la idoneidad de la propuesta en términos de participación y motivación del alumnado.

Se trata de un diseño de investigación de corte cualitativo, exploratorio y cuasi experimental (Babbie, 2005; McMillan y Schumacher, 2005) basado en el análisis de la forma de actuar de los participantes seleccionados ante una propuesta de intervención con fuentes objetuales, donde el investigador jugaría un papel activo durante el proceso. Para ello, se llevó a cabo un diseño con un único grupo con pretest/postest que permitiría analizar los resultados de aprendizaje tras la implementación de una intervención educativa creada ad hoc. La investigación se cerró con el análisis de la propia valoración del estudiante respecto a la propuesta llevada a cabo en el aula. 


\subsection{Participantes y contexto}

El método de selección de la muestra de la presente investigación fue un muestreo no probabilístico de tipo intencional (Babbie, 2000). El centro participante fue un centro público de Educación Infantil de la Región de Murcia, situado en una ciudad de la comarca natural del Bajo Guadalentín. Esta localidad alberga una gran riqueza cultural y cuenta con un patrimonio extenso, ya que es una localidad en la que han convivido diferentes culturas, cuyo legado histórico y cultural queda recogido en el museo arqueológico de la localidad. Es por ello que se decidió utilizar el patrimonio cultural de la zona como hilo conductor de la intervención creando de este modo aprendizajes significativos y cercanos a los participantes.

La intervención se enfocó en un grupo natural en el contexto escolar como es el grupo-clase. Concretamente contamos con un total de 19 alumnos de 5 y 6 años, donde 10 de ellos eran niñas y 9 eran niños. El alumnado participante sobre el que se realizó la intervención no había tenido experiencia previa con el contacto y análisis de fuentes en el aula (datos obtenidos a través de una entrevista previa a la docente). El contacto que hasta el momento había tenido el alumnado participante con la historia se caracterizaba por la escasa presencia de contenidos históricos y el trabajo de proyectos aislados que en principio no guardaban relación con el entorno del niño. Esta situación se mostraba similar a la detectada en la investigación llevada a cabo por Casanova, Arias y Egea (2018b) donde, a pesar de que los centros son más proclives a utilizar alternativas metodológicas como el método por proyectos, éstos quedan asociados a editoriales que imponen los contenidos a trabajar (en su mayoría Egipto, los castillos de la Edad Media y la Prehistoria) obviando el contexto propio del alumnado y la riqueza patrimonial e histórica de los mismos.

\subsection{Instrumentos utilizados para la recogida de información}

Esta investigación se basó en la observación participante en el aula, a través de notas de campo, y se completó con el uso de técnicas indirectas de recogida de información, como las grabaciones en vídeo y en audio (Bisquerra, 2014). El análisis se centró especialmente en los resultados obtenidos en el pretest/postest y en la escala de valoración realizada a los propios estudiantes.

El pretest permitió conocer el punto de partida de los alumnos antes de la intervención. Para su realización se presentó a los alumnos una serie de objetos que debían analizar autónomamente. La única consigna que se les daba era que debían decir todo lo que consideraran sobre el objeto seleccionado.

El postest permitió conocer los conocimientos y habilidades alcanzadas o adquiridas por el alumnado tras realizar las tres sesiones de intervención específica con fuentes objetuales. En este, el alumno debía analizar una serie de objetos de forma completamente autónoma, esperando como resultado que los participantes aplicasen lo trabajado durante las sesiones de intervención anteriores. La comparación de los resultados obtenidos tras la aplicación de ambos instrumentos dio respuesta a los objetivos 1-3.

Por último, para dar respuesta al objetivo 4, se utilizó una escala de valoración basada en la denominada técnica del semáforo. Esta consiste en que los alumnos deben mostrar su opinión con 
respecto a la propuesta en términos de motivación a través de un código de color que representan las siguientes valoraciones: una carita verde indica que "me ha gustado", una carita amarilla señala que "me ha gustado regular", y una carita roja equivale a "no me ha gustado". Los alumnos debían de elegir una e introducirla en un buzón diseñado para tal fin, debiendo añadir verbalmente el porqué de su elección.

\subsection{Propuesta de intervención}

La intervención llevada a cabo fue titulada con el nombre Tras la pista de los romanos en mi ciudad. El hilo conductor de la propuesta fue el mundo romano, por la importancia y la presencia que éste tiene en el patrimonio local. La historia comenzaba con una supuesta excavación en la localidad, realizada por una arqueóloga que llamó a la colaboración del alumnado participante para analizar los objetos hallados en dos escenarios diferentes: los baños romanos, situados en el museo arqueológico, y una villa romana cuyos restos aparecieron en la plaza de la iglesia de la misma localidad.

La intervención contó con seis sesiones: motivación, pretest, tres sesiones de intervención específica y postest (Tabla 2), con una duración de 45 minutos cada una. Todas las sesiones siguieron la misma organización. Los niños, sentados en la asamblea, primero observaban los objetos de uno en uno y los tocaban. Después, se les realizaban una serie de preguntas para analizar las fuentes, que debían de responder levantando la mano. La intervención estuvo basada pues en el diálogo a través de preguntas y respuestas y en la manipulación y observación de los objetos, de ahí la importancia del registro audiovisual como técnica de recogida de datos.

Tabla 2

Temporalización y descripción de las diferentes sesiones destinadas a la intervención

\begin{tabular}{|c|c|c|}
\hline Sesión & Contenidos y tareas a desarrollar & Acciones asociadas \\
\hline $\begin{array}{l}1 \text { Motivación/ } \\
\text { presentación }\end{array}$ & $\begin{array}{l}\text { Conocimientos previos sobre: } \\
\text { civilización romana, arqueología, } \\
\text { museo de la localidad }\end{array}$ & Motivación e ideas previas \\
\hline 2 Pretest & $\begin{array}{l}\text { Realización pretest. Primeras } \\
\text { reacciones frente a los objetos }\end{array}$ & $\begin{array}{l}\text { Observación y manipulación de objetos } \\
\text { Realización de inferencias de forma autónoma }\end{array}$ \\
\hline 3 Intervención 1 & $\begin{array}{l}\text { Análisis de aspectos formales de las } \\
\text { fuentes objetuales, planteamiento de } \\
\text { hipótesis y conclusiones }\end{array}$ & $\begin{array}{l}\text { Observación y manipulación de objetos } \\
\text { Enseñanza del proceso de análisis de objetos }\end{array}$ \\
\hline 4 Intervención 2 & $\begin{array}{l}\text { Análisis de aspectos formales de las } \\
\text { fuentes objetuales, planteamiento de } \\
\text { hipótesis y conclusiones }\end{array}$ & $\begin{array}{l}\text { Observación y manipulación de objetos } \\
\text { Realización de inferencias con ayuda }\end{array}$ \\
\hline 5 Intervención 3 & $\begin{array}{l}\text { Análisis de aspectos formales de las } \\
\text { fuentes objetuales, planteamiento de } \\
\text { hipótesis y conclusiones }\end{array}$ & $\begin{array}{l}\text { Observación y manipulación de objetos } \\
\text { Realización de inferencias de forma autónoma, } \\
\text { recordando las pautas solo si necesitan ayuda }\end{array}$ \\
\hline $\begin{array}{l}6 \text { Postest/ } \\
\text { conclusión }\end{array}$ & $\begin{array}{l}\text { Realización postest } \\
\text { Evaluación por parte del alumnado }\end{array}$ & $\begin{array}{l}\text { Observación y manipulación de objetos } \\
\text { Realización de inferencias de forma autónoma }\end{array}$ \\
\hline
\end{tabular}

Fuente: elaboración propia. 
$\mathrm{Al}$ estar la intervención basada en los hallazgos producidos en una villa romana, los objetos debían estar relacionados con distintas dependencias (Tabla 3). Se consideró adecuado incluir tres objetos por sesión para poder recoger la información necesaria. Con respecto al orden de presentación, se decidió iniciar el análisis en cada sesión con el objeto más fácil o reconocible y finalizar con el que presentaba mayores dificultades. Otro aspecto a tener en cuenta fue no dar las respuestas correctas hasta que no se hiciese el análisis de todos los objetos trabajados en la sesión, evitando que las respuestas incorrectas concluyeran en sentimientos de incompetencia o frustración.

Tabla 3

Fuentes objetuales utilizadas durante las distintas sesiones de la propuesta de investigación y preguntas trabajadas con cada una de ellas

\begin{tabular}{|c|c|c|}
\hline Sesión & Objetos & Preguntas asociadas a los objetos \\
\hline 1 & - & \multirow{3}{*}{$\begin{array}{l}\text { Aspectos formales } \\
\text { ¿Cómo es?, ¿qué forma tiene? ¿De qué material está } \\
\text { hecho? ¿Está decorado?, ¿de qué color es?, ¿tiene dibujos } \\
\text { o letras? }\end{array}$} \\
\hline 2 & Pre-test: esponja, monedas, lucerna & \\
\hline 3 & Cuchara de madera, vasito, mortero & \\
\hline 4 & Muñeca, caballo, tablillas & \multirow{3}{*}{$\begin{array}{l}\text { Hipótesis } \\
\text { ¿Qué es?, ¿para qué pudo servir?, ¿cómo lo sabéis? ¿A } \\
\text { quién pudo pertenecer?, ¿cómo lo sabéis? ¿Piensas que es } \\
\text { un objeto importante? }\end{array}$} \\
\hline 5 & Aguja para el pelo, espejo, llave & \\
\hline 6 & ungüentario, dedal, & \\
\hline
\end{tabular}

Fuente: elaboración propia a partir de Egea, Pernas y Arias (2014).

\subsection{Plan de análisis de la información}

Recordamos que la metodología de investigación fue de carácter cualitativo ya que se pretendía analizar la interacción verbal del alumnado. Es por ello que las respuestas han sido siempre de carácter abierto y flexible. Las interacciones observadas en las sesiones de pretest y postest fueron transcritas, categorizadas y codificadas a través del programa informático Atlas.ti, versión 8, que facilitó la creación de redes semánticas atendiendo a cada una de las unidades principales de análisis creadas (coincidentes con los objetivos de investigación 1-3 planteados): (1) observación, (2) interacción y (3) establecimiento de hipótesis.

Estas unidades de análisis han sido a su vez divididas en categorías, atendiendo a las preguntas formuladas, y subcategorías, atendiendo a las respuestas obtenidas (tablas 4, 5 y 6). Estas respuestas del alumno (o subcategorías) han sido clasificadas en dos niveles: si el estudiante hacía uso de un punto de vista individual basado en su experiencia cercana, sería un nivel básico (representado en color rojo en el conjunto del análisis); si, por el contrario, era capaz de aplicar los conocimientos y destrezas adquiridas, este sería considerado un nivel óptimo (y será representado con el color verde). La codificación en estas dos tonalidades permite mostrar de una forma más visual el avance del niño desde la aplicación exclusiva de su experiencia directa (color rojo) hacia la aplicación de nuevos conocimientos (color verde), por lo que será la codificación de color utilizadas en las figuras 1-6, donde se exponen los resultados obtenidos. 
Tabla 4

Categorías y subcategorías utilizadas en el análisis cualitativo del objetivo 1 (observación)

\begin{tabular}{|c|c|c|c|}
\hline Categoría & Subcategoría & Descripción & Nivel \\
\hline \multirow[t]{4}{*}{ Forma } & Función & Relación entre la forma y la función del objeto & Básico \\
\hline & Forma & Relación con objetos de apariencia similar & \\
\hline & F. geométrica/tamaños & Establecimiento de formas geométricas y tamaños & Óptimo \\
\hline & Grosor & Establecen distintos tipos de grosor & \\
\hline \multirow[t]{4}{*}{ Material } & Forma & Relacionan el material con objetos de apariencia similar & Básico \\
\hline & Olor & Atribuyen olores a los objetos & Óptimo \\
\hline & Textura & Atribuyen texturas a los objetos & \\
\hline & Material & Atribuyen el material al objeto & \\
\hline \multirow[t]{4}{*}{ Decoración } & Forma & $\begin{array}{l}\text { Relacionan objetos con formas similares pare describir la } \\
\text { decoración }\end{array}$ & Básico \\
\hline & Detalles & Describen detalles de los objetos & Óptimo \\
\hline & Colores & Establecen colores & \\
\hline & Estado del objeto & Establecen estados del objeto & \\
\hline
\end{tabular}

Fuente: elaboración propia

Tabla 5

Categorías y subcategorías utilizadas en el análisis cualitativo del objetivo 2 (interacción)

\begin{tabular}{lllc}
\hline \multicolumn{1}{c}{ Categoría } & \multicolumn{1}{c}{ Subcategoría } & \multicolumn{1}{c}{ Descripción } & Nivel \\
\hline $\begin{array}{l}\text { Inicio de la } \\
\text { interacción }\end{array}$ & Al coger el objeto & El participante da una respuesta al coger el objeto & Básico \\
& $\begin{array}{l}\text { Durante la } \\
\text { interacción } \\
\text { Tras la interacción }\end{array}$ & $\begin{array}{l}\text { El participante da una respuesta al mismo tiempo } \\
\text { que interactúa con el objeto } \\
\text { Tras haber interactuado con el objeto el participante } \\
\text { da una respuesta }\end{array}$ & Óptimo \\
\hline $\begin{array}{l}\text { Tipo de } \\
\text { interacción }\end{array}$ & $\begin{array}{l}\text { Solo observación } \\
\text { Representación }\end{array}$ & $\begin{array}{l}\text { Únicamente observa } \\
\text { Representa simbólicamente la respuesta }\end{array}$ & Básico \\
\hline Simbólica & Exploración & Interactúa con el objeto de forma más profunda, & \\
Tiempo de & Escaso & La interacción dura de 0-2 segundos & Básico \\
\hline interacción & Medio & La interacción dura de 3-5 segundos & Óptimo \\
\hline
\end{tabular}

Fuente: elaboración propia.

En cuanto a la codificación seguida para mantener el anonimato de los participantes, se estableció para cada uno de ellos un número correlativo del 1 al 19, que fue mantenido a lo largo de todo el proceso. A partir de ahí, se utilizó la letra A para hacer referencia a las aportaciones realizadas por el mismo individuo en el pretest (A1-A19) y la letra B para las aportaciones o comentarios realizados en el postest (B1-B19). 
Tabla 6 Categorías y subcategorías utilizadas en el análisis cualitativo del objetivo 3 (establecimiento de hipótesis)

\begin{tabular}{lllc}
\hline Categoría & Subcategoría & Descripción & Nivel \\
\hline Función & Forma & Relación entre la forma y la función del objeto & Básico \\
\cline { 2 - 4 } & Función & Establecen una función real a partir de la experimentación & Óptimo \\
\hline Pertenencia & $\begin{array}{l}\text { Rol no } \\
\text { contextualizado } \\
\text { Rol contextualizado }\end{array}$ & $\begin{array}{l}\text { No aplican ningún tipo de perspectiva histórica } \\
\text { pertenecer }\end{array}$ & Básico \\
\cline { 3 - 4 } & & & Óptimo \\
\hline
\end{tabular}

Fuente: elaboración propia.

\section{Resultados}

El objetivo 1, centrado en analizar la capacidad de observación del alumnado a través de preguntas sobre los objetos, se centra en la unidad de análisis observación y las categorías forma, material y decoración (Tabla 4). El conjunto de menciones alusivas a esta unidad de análisis realizadas por el alumnado participante en el pretest fue $\mathrm{N}=53$ (Figura 1).

En relación a la forma de los objetos, se contabilizaron 38 menciones, utilizando mayoritariamente su experiencia como guía de la descripción (n=33). Es decir, el alumnado participante describía el objeto a partir de otros objetos que compartían forma (n=27) o función $(n=6)$. Menos frecuente fue la descripción atendiendo a contenidos específicos de la etapa $(n=5)$.

Siguiendo con la mención al material del que estaba realizado el objeto, el alumnado realizó observaciones al respecto en 10 ocasiones. Al igual que con la forma, utilizó su experiencia como guía de la descripción ( $\mathrm{n}=9$ ) al describir el objeto a partir de otros que compartían forma similar. Menos frecuente fue la descripción atendiendo a contenidos específicos de la etapa (n=1).

En cuanto a la decoración, el alumnado realizó observaciones al respecto en 5 ocasiones. En esta ocasión primó la aplicación de conocimientos como guía de la descripción (n=4): los participantes describían el objeto atendiendo a los detalles que en ellos encontraban y centraban la atención en el objeto en sí.

En relación al postest, y en base al análisis de las mismas categorías (forma, material y decoración) se contabilizaron un total de 66 menciones (N=66) (Figura 2).

El alumnado realizó observaciones respecto a la forma del objeto en 30 ocasiones. Pese a que el alumnado participante describió el objeto a partir de otros que compartían forma (n=9) o función ( $n=6)$, existió un aumento de observaciones en las que el alumno mencionó formas geométricas y tamaños $(n=13)$ y grosores $(n=2)$, aplicando los conocimientos adquiridos en la etapa.

En relación al material, el alumnado realizó un total de 10 menciones, todas ellas relacionadas con la aplicación de conocimientos $(n=10)$. El alumnado participante describió los materiales de los objetos usando los nombres de materiales específicos (n=8) y, en algunos casos, aunque en menor medida, mencionó los olores $(\mathrm{n}=2)$.

En cuanto a la decoración, el alumnado realizó observaciones al respecto en 26 ocasiones. En este caso, los participantes describieron el objeto atendiendo a los diferentes detalles que en 
ellos encontraban $(n=15)$ e hicieron mención a los colores $(n=8)$ y a características relacionadas con el estado de conservación del objeto $(n=3)$.

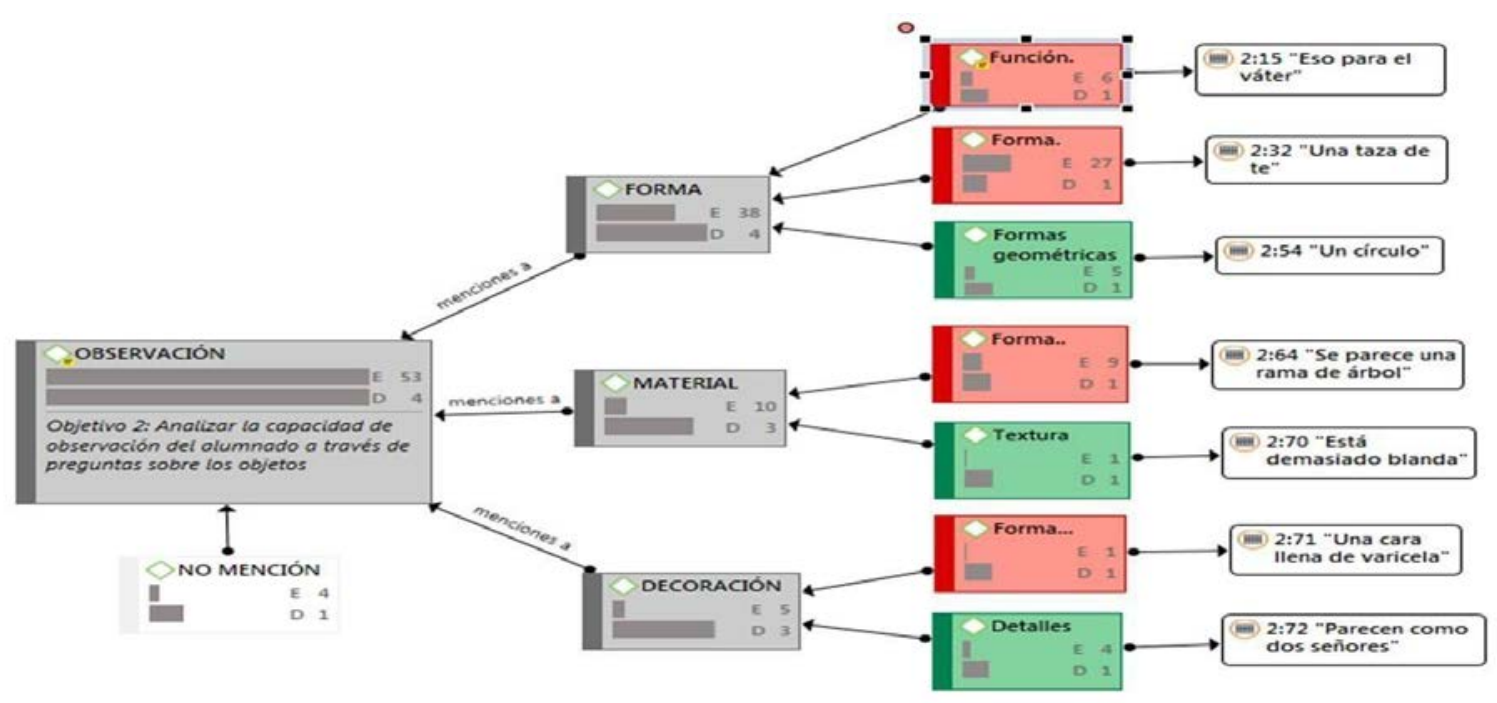

Figura 1. Resultados en relación a la observación de los objetos en la fase pretest.

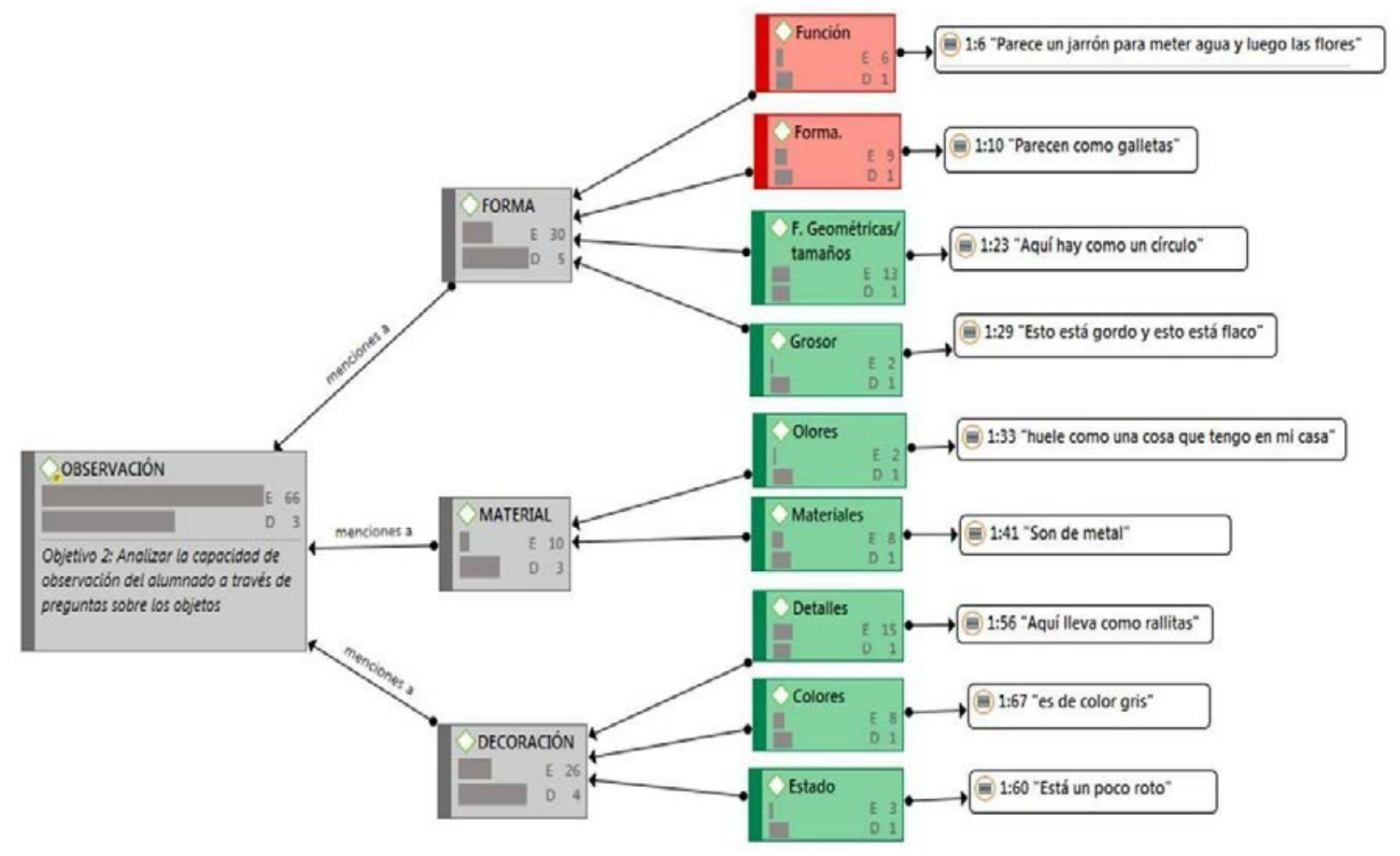

Figura 2. Resultados en relación a la observación de los objetos en la fase postest.

Para dar respuesta al objetivo 2, conocer el grado de interacción que los alumnos establecen a la hora de manipular, observar y analizar las fuentes objetuales, nos centramos en la unidad de análisis interacción y en las categorías: inicio, tipo y tiempo de interacción (Tabla 5). El conjunto de interacciones analizadas en el pretest y en el postest fue de 57 (N=57): una interacción por niño y objeto (19 participantes, 3 objetos) (Figura 3). 
Centrándonos en los datos del pretest, y en relación al inicio de la interacción verbal, el alumnado participante inició mayoritariamente la interacción verbal nada más coger el objeto $(n=35)$. En un porcentaje menor, los alumnos iniciaron la interacción verbal tras la interacción con el objeto $(n=13)$ o durante la misma $(n=9)$. En cuanto al tipo de interacción, el alumnado participante interactuó con el objeto únicamente a través de la mera observación $(n=40)$. Menos frecuente fue la interacción de forma exploratoria $(n=12)$ y solo unos pocos se apoyaron en la representación simbólica a la hora de utilizar e interactuar con el objeto $(n=5)$. Finalmente, en relación al tiempo de interacción con el objeto, los participantes interactuaron con el objeto en un tiempo considerado medio $(n=25)$ y escaso $(n=19)$. En menor medida, los participantes interactuaron con el objeto un tiempo considerado prolongado, que sería lo suficiente y adecuado para que el alumno realizara una interacción completa que le permita estudiar el objeto $(n=13)$.

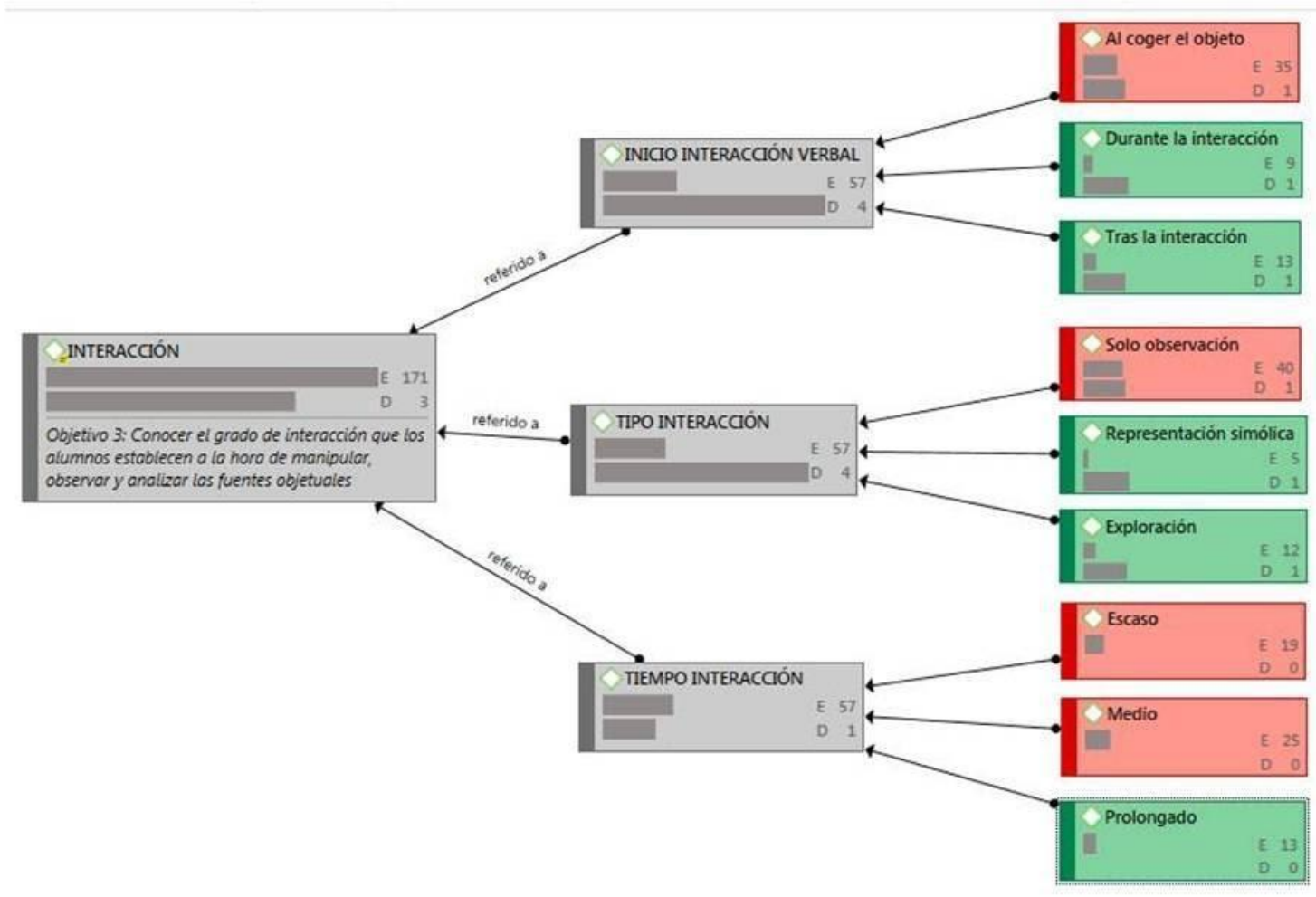

Figura 3. Resultados en relación a la interacción con los objetos en la fase pretest.

En cuanto al postest (Figura 4), el alumnado inició la interacción verbal mayoritariamente durante el contacto con el objeto $(n=34)$ y en menor medida tras la interacción con el objeto $(n=23)$. En ningún caso dió la respuesta antes o al inicio de la interacción con el objeto. En cuanto al tipo de interacción, el alumnado lo hizo a través de la exploración de los mismos (n=47) y algunos lo hicieron a través de la representación simbólica $(n=10)$. En ningún caso el alumnado se limitó únicamente a la observación somera del mismo.

Por último, atendiendo al tiempo que el alumno interactuó con el objeto, la mayoría lo hicieron durante un tiempo considerado prolongado $(n=38)$ y menos durante un tiempo considerado medio $(n=19)$. 


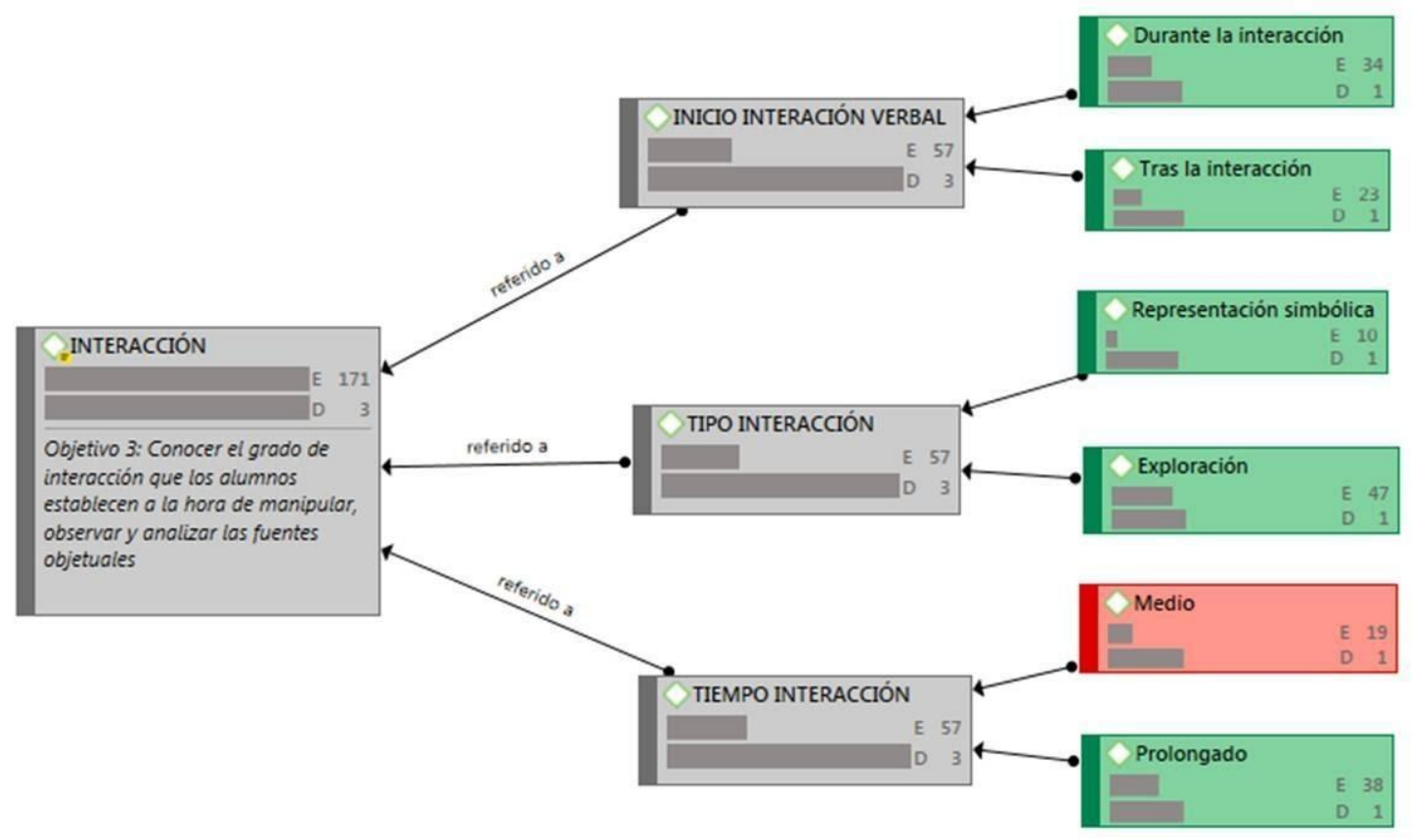

Figura 4. Resultados en relación a la interacción con los objetos en la fase postest.

Por último, para dar respuesta al objetivo 3, valorar la capacidad de utilizar fuentes objetuales para realizar hipótesis sencillas y establecer conclusiones, se ha analizado la unidad establecimiento de hipótesis y las categorías: función y pertenencia (Tabla 6). En este sentido, el conjunto de menciones alusivas a la realización de hipótesis en el pretest fue de $53(\mathrm{~N}=53)$ (Figura 5). En relación a la función de los objetos seleccionados, el alumnado participante realizó hipótesis en las que relacionaba la función con la forma de objetos con los que encontraba similitud $(n=47)$. En menor medida el alumno consiguió realizar hipótesis a partir de la experimentación con el mismo $(n=6)$.

En relación a quién o quiénes pudieron pertenecer los objetos, el alumnado no realizó mención alguna al respecto $(n=0)$. También resulta conveniente destacar que en el pretest, en 4 ocasiones $(\mathrm{N}=4)$, los alumnos no realizaron ningún tipo de intervención.

En el postest se registraron menos menciones asociadas a la elaboración de hipótesis $(\mathrm{N}=33)$, y estuvieron centradas todas ellas en la función de los objetos (Figura 6). En este caso, destacaron las respuestas asociadas a la aplicación de conocimientos, donde el alumno dedujo la funcionalidad del objeto después de experimentar con él $(n=25)$, siendo menos frecuente la aparición de respuestas limitadas a la observación somera del mismo (n=8). 


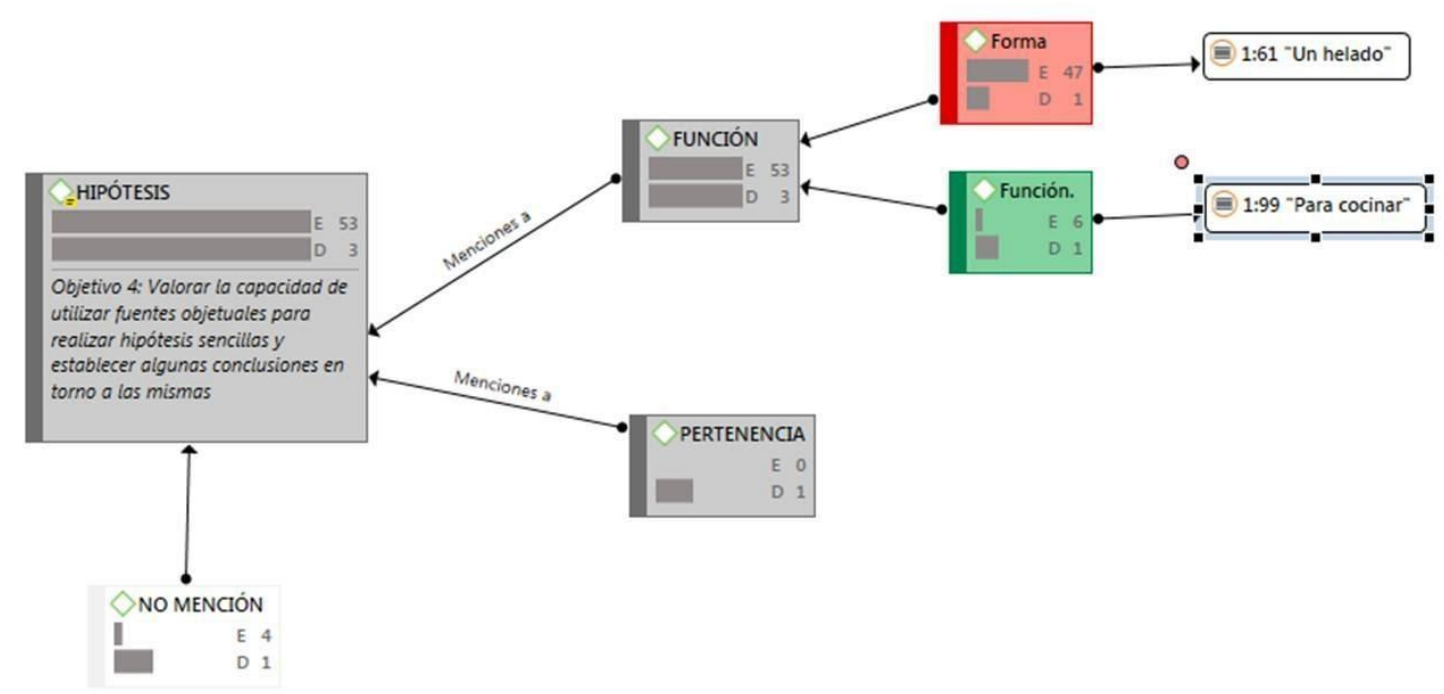

Figura 5. Resultados en relación a la realización de hipótesis sobre los objetos en la fase pretest.

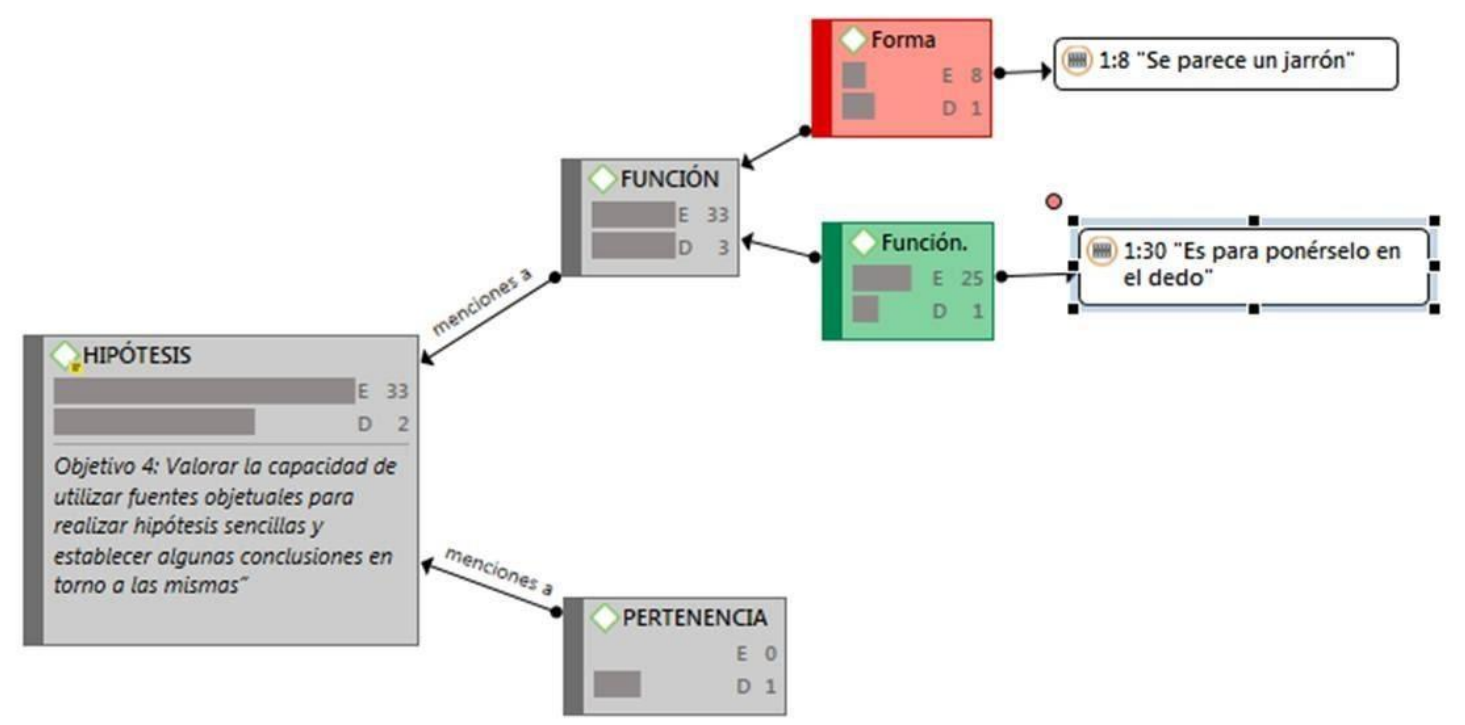

Figura 6. Resultados en relación a la realización de hipótesis sobre los objetos en la fase postest.

Pese a que no se ha producido un aumento considerable de esta categoría, sí se produjeron cambios a la hora de expresar sus ideas e hipótesis. Recordamos que toda la propuesta se basó en la aplicación del método dialógico, pues lo que se pretendió fue analizar las respuestas orales y gestuales de los participantes a partir de la interacción en grupo. Los estudiantes pasaron del uso de una o dos palabras en sus respuestas a estructuras que unían dos o tres frases breves. Es decir, los estudiantes crearon estructuras algo más elaboradas. Pese a esto, el conector más utilizado fue "y". En muy pocas ocasiones el alumno utilizó otro tipo de nexos debido a la inmadurez del lenguaje y a que posiblemente se trabaje poco la argumentación de respuestas en clase.

$[10 \mathrm{~A}]$ "una tetera"

[10B] "Que aquí hay un agujero y aquí otro para poner las flores, y aquí uno grande y aquí como un círculo" 
Por último, en relación al objetivo 4, evaluar la idoneidad de la propuesta en términos de participación y motivación del alumnado, se obtuvieron los siguientes niveles de respuesta: me ha gustado $(\mathrm{n}=22)$, me ha gustado regular $(\mathrm{n}=1)$ y no me ha gustado $(\mathrm{n}=0)$ (Figura 7). Preguntados los estudiantes expresamente sobre su opinión, estos destacaron los nuevos aprendizajes adquiridos $(n=12)$ y la experiencia de haber manipulado objetos $(n=10)$. Solo hubo una valoración regular $(\mathrm{n}=1)$, donde la alumna expresamente señaló que la intervención había resultado corta y que le hubiera gustado trabajar más.

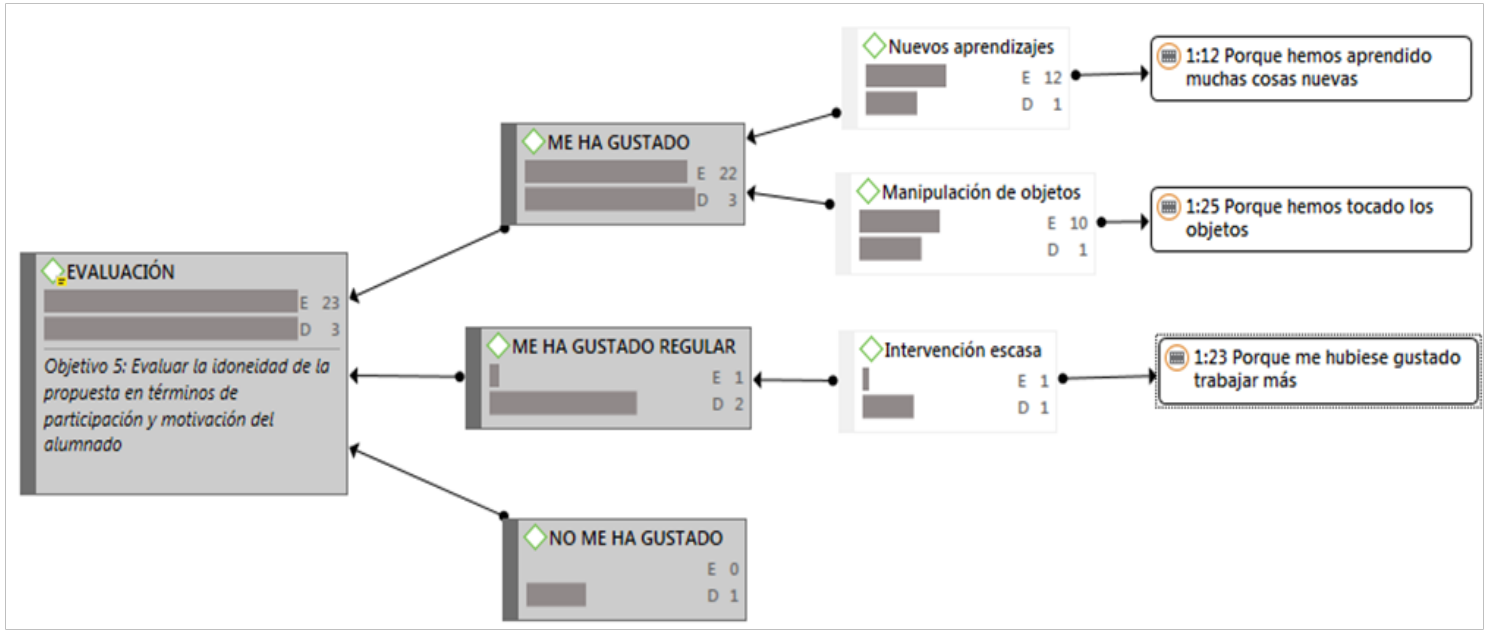

Figura 7. Resultados en relación a la motivación del alumnado (objetivo 4).

\section{Discusión e interpretación de los resultados}

A pesar de que los discentes no han tenido experiencias previas con el trabajo de fuentes objetuales, ni de cualquier otro tipo, se ha observado una evolución positiva en prácticamente todas las habilidades que se pretendían observar y que nos permiten ser optimistas en cuanto a la posibilidad de desarrollar habilidades específicas asociadas al pensamiento histórico a través del uso de fuentes. En relación al trabajo de un método, el alumnado se preocupó de seguir los pasos establecidos en el análisis de fuentes objetuales: observación, manipulación y realización de hipótesis.

En un primer momento, el alumnado, ante el contacto con las fuentes, ofreció una respuesta rápida y final, saltándose los pasos previos de análisis y contacto con las fuentes. Tras el desarrollo de tres intervenciones específicas, el conjunto del alumnado aplicó un método basado en la observación y descripción como paso previo al establecimiento de cualquier tipo de respuesta. Es en el proceso de observación donde pudimos comprobar una enorme diferencia entre el pretest y el postest. En el primer contacto establecido con las fuentes el conjunto del alumnado presentó muchas dificultades para describir los aspectos morfológicos de las fuentes utilizadas, una tarea relativamente sencilla y que entra dentro de los contenidos específicos de la etapa. A pesar de ello, la mayoría de respuestas atendieron únicamente a un atributo del objeto, mezclando la fantasía y la experiencia previa. Tras haber trabajado esta habilidad en las sesiones previas al postest, se pude comprobar en este cómo la observación pasó a ser más meticulosa, y el alumnado comenzó a tener en cuenta diferentes atributos de los objetos. El alumnado pasó a fijar la mirada en el objeto y no en la docente: 
$[3 \mathrm{~A}]$ "una moneda"

[3B] "parece que son monedas y tiene dibujitos y esto es de color amarillo y estos gris y estos son grandes y esas pequeñas"

En relación a las habilidades de interacción, también se pudieron observar cambios considerables. En un primer momento, la interacción que los alumnos establecieron con las fuentes era escasa ya que cogían los objetos con miedo, de forma rígida, posándolos sobre las manos sin llegar a manipularlos, hecho que es entendible ya que nunca se había trabajado con fuentes históricas en el aula. Tras la puesta en práctica de la intervención, se vio un cambio notable en el que el alumnado comenzó a realizar una interacción real con los objetos, los manipuló, los olió, los agitó, los puso sobre el suelo. También hay que tener en cuenta que el alumno dejó de ofrecer una respuesta sin haber interactuado previamente con el objeto. Este apreció pues cómo la manipulación resultaba clave para dar su respuesta. El alumnado pasó a ofrecer respuestas más meditadas fruto de una observación más meticulosa.

Junto a esto, el alumnado participante fue capaz de realizar hipótesis sencillas, aunque centradas solo en el primer estadio relacionado con la funcionalidad de los objetos. Además, se produjeron cambios a la hora de expresar sus ideas e hipótesis, aunque todavía se destaca la ausencia de nexos complejos que expresen posibilidad.

\author{
[6A] “Una esponja” \\ [6B] "Parece un dedal. Tiene puntitos y es como para ponérselo en el dedo y no \\ les pinchen"
}

Sin embargo, el alumnado no ha conseguido llegar a realizar hipótesis que requerían de mayor abstracción, como es en relación a quiénes pudieron pertenecer las fuentes objetuales. Aun siendo guiados por el investigador, las respuestas eran confusas y llenas de generalidades. Investigaciones realizadas en contextos similares con imágenes sí que obtuvieron un mayor nivel de inferencia de los estudiantes, si bien se apreció que el desarrollo de la expresión asociada a la formulación de hipótesis todavía no estaba completamente desarrollada y se basaba principalmente en la entonación y no en el uso de conectores (Arias et al., 2019).

De esto se deduce que, una mayor incidencia de este tipo de metodologías en el aula, derivarían previsiblemente en una mayor autonomía del alumnado en este sentido, como así lo avalan, junto a esta, otras investigaciones como las de Harnett y Whitehouse (2013) y Sewar y Boertien (2004). En ambas los participantes aprendieron a pensar y a comunicarse como historiadores, mejoraron en la habilidad de observación, exploración y en el trabajo a partir de un método de investigación, y se consiguió una alta participación y el entusiasmo ante el contacto con fuentes. Es por ello, que no se debe de pasar por alto la necesidad de enseñar a los alumnos el método científico como base para el aprendizaje de la historia a través de estrategias activas, analíticas y reflexivas, ya que lo que se pretende es que los discentes resuelvan auténticos problemas históricos. Recordamos que la utilización de pruebas está en la base de todo conocimiento científico (Cooper, 2002; Seixas y Morton, 2013). 


\section{Conclusiones}

En este estudio se ha querido valorar el aprendizaje resultante de la implementación de una experiencia basada en la didáctica del objeto en un aula de cinco años y su relación con el desarrollo de habilidades de observación, interacción y establecimiento de hipótesis. Tal y como se ha podido comprobar tras el análisis de los resultados, la didáctica del objeto inicia al alumnado en el conocimiento de habilidades propias del método científico que permiten analizar diferentes fuentes objetuales, además de promover una motivación por la historia por el hecho de poder "tocarla".

Algunas de las posibilidades e implicaciones educativas que suponen el uso de fuentes primarias en el aula de Educación Infantil, y coincidiendo con autores como Santacana y Llonch (2012) y Arias y Casanova (2018a), se pueden destacar las siguientes:

- El objeto convierte las relaciones pasado-presente en algo tangible: se pasa de una relación abstracta a un instrumento perceptible a través de los sentidos.

- Los objetos permiten introducir y aplicar una gran diversidad de contenidos asociados a la propia etapa en la que se encuentra el niño en un contexto dotado de significado y propósito.

- Las fuentes nos permiten introducir el trabajo con fuentes con alumnos de edades tempranas que aún no tienen adquirida la capacidad lectora a partir de preguntas y diálogo, fundamental para la construcción de conocimiento.

- Los objetos se muestran como un recurso ideal para iniciar al alumnado en el método científico y establecer hipótesis e inferencias.

- Los objetos aumentan el interés ante el conocimiento de la historia y la participación de los discentes.

Pero pese a los beneficios y ventajas enumeradas y obtenidas tras la implementación de la propuesta, todavía es escasa y poco común la incorporación de fuentes en el aula para el tratamiento de las habilidades históricas (Morales, Egea y Arias, 2017), a pesar de las aportaciones y recomendaciones ofrecidas por diferentes autores (Ashby, 2011; Cooper, 2002; Santacana y Llonch, 2012; Seixas y Morton, 2013). Es por ello que se debe promover un cambio en la enseñanza de la historia y abandonar de una vez por todas la visión tradicional de memorización de datos y hechos históricos, que también se produce en la etapa infantil, y que conducen a un fracaso en etapas posteriores, condenando a la disciplina a ser una materia aburrida y obsoleta. Tal y como afirman Seixas y Morton (2013), "hacer historia debe ser divertido y serio, difícil y gratificante, significativo y creativo" (p. 9). Debemos dejar pues al alumno mirar, tocar, pensar y expresar para poder construir y dotar a la historia de un significado para el estudiante.

\section{Referencias bibliográficas}

Arias, L. y Casanova, E. (2018). El uso de los objetos arqueológicos en las aulas de educación infantil: una experiencia en un aula de cuatro años. En A. Egea, L. Arias y J. Santacana 
(Coords.), Y la arqueología llegó al aula. La cultura material y el método arqueológico para la enseñanza de la historia y el patrimonio (pp. 121-135). Gijón, España: Trea.

Arias, L., Casanova, E., Egea, A., García, A. B. y Morales, M. J. (2016). Aprendiendo a tocar la historia. Las fuentes objetuales como recurso de aprendizaje en Educación Infantil y Primaria. En R. López-Facal (Ed.), Ciencias sociales, educación y futuro. Investigaciones en didáctica de las ciencias sociales. Actas del VII Simposio de Didáctica de las Ciencias Sociales en el ámbito Iberoamericano (pp. 136-148). Santiago de Compostela: Red 14-Universidad de Santiago de Compostela.

Arias-Ferrer, L., Egea-Vivancos, A., \& Levstik, L. S. (2019). Historical Thinking in the Early Years: The Power of Image and Narrative. En K. J. Kerry-Moran \& J.-A. Aerila (Eds.), Story in Children's Lives: Contributions of the Narrative Mode to Early Childhood Development, Literacy, and Learning (pp. 175-198). Springer International Publishing.

Ashby, R. (2011). Understanding historical evidence. En I. Davies (Ed.), Debates in history teaching (pp. 137-147). New York, Routledge.

Babbie, E. (2000). Fundamentos de la investigación social. Madrid, España: International Thomson. Bisquerra, R. (2014). Metodología de la investigación educativa. Madrid: La Muralla.

Bruner, J. (1999). The process of Education (1st ed. 1960). Cambridge, USA: Harvard University Press.

Casanova, E., Arias, L. y Egea, A. (2018). La metodología por proyectos como oportunidad para la introducción de la historia y el patrimonio en las aulas de educación infantil, Contextos educativos, 22, 79-95. Doi: 10.18172/con.3185

Cooper, H. (2002). History in the early years. Londres-New York: Rutledge.

Cooper, H. (ed.) (2013). Teaching history creatively. Oxon-Nueva York: Routledge.

De Groot-Reuvekamp, M. J. D., Boxtel, C. V., Ros, A. y Harnett, P. (2014). The understanding of historical time in the primary history curriculum in England and the Netherlands. Journal of Curriculum Studies, 46(4), 487-514. https://doi.org/10.1080/00220272.2013.869837

Dean, J. (2008). Ensenyar història a primària. Cambridge, Reino Unido: Zenobita.

Egea, A., Pernas, S. y Arias, L. (2014). Re- construyendo la historia a partir del patrimonio arqueológico. En O. Fontal, A. Ibáñez y L. Martín (Coords.), Reflexionar desde las experiencias. Una visión complementaria entre España, Francia y Brasil. Actas del II congreso internacional de educación patrimonial (pp. 1149-1157). Madrid: IPCE/OEPE.

Harnett, P. (1993). Identifying progression in children's understanding: The use of visual materials to assess. Cambridge Journal of Education, 23(2), 137.

Harnett, P. y Whitehouse, S. (2013). Investigating activities using sources. En H. Cooper (Ed), Teaching history creatively (pp. 29-50). Oxon-Nueva York: Routledge.

Lee, P. y Ashby, R. (2000). Progression in historical understanding among students ages 7-14. En P. N. Stearns, P. Seixas y S. Wineburg. (Eds.) Knowing, teaching and learning history (pp. 223245). Nueva York, NY-Londres, UK: New York University Press.

Levesque, S. (2008). Thinking historically. Educating students for the twenty-first century. TorontoBuffalo, Nueva York, NY-Londres, UK: University of Toronto Press. 
Levstik, L. S., y Barton, K. C. (2015). Doing history. Investigating with children in elementary and middle schools (5. a ed.). Nueva York, NY: Routledge.

Maine, F. (2015) Dialogic Readers: Children talking and thinking together about visual texts. Florence, USA: Taylor and Francis.

McMillan, J. y Schumacher, S. (2005). Investigación educativa. Madrid, España: Pearson.

Miralles, P. y Rivero, P. (2012). Propuesta de innovación para la enseñanza de la historia en Educación Infantil. REIFOP, 15(1), 81-90.

Morales, M. J., Egea, A. y Arias, L. (2017). El patrimonio y las fuentes materiales en la práctica docente de la Educación Primaria en la Región de Murcia. Her\&Mus, 18, 102-115.

Pappas, C., Kiefer, B. Z., \& Levstik, L. S. (2006). An integrated language perspective in the elementary school: An action approach (4th ed). Pearson Allyn and Bacon.

Prats, J. y Santacana, J. (2011). Métodos para la enseñanza de la historia. En Prats (Coord.), Didáctica de la geografía y la historia (pp. 51-64). Barcelona, España: Graó.

Santacana, J. y Llonch, N. (2012). Manual de didáctica del objeto en el museo. Gijón: Trea.

Seixas, P. y Morton, T. (2013). The big six historical thinking concepts. Toronto: Nelson Education.

Seward, D., y Boertien, V. (2004). All about us. En H. Cooper (Ed.), Exploring time and place trough play (pp. 24-39). Londres, Gran Bretaña: David Fulton Publishers.

Solé, G. (2009). A história no 1o ciclo do Ensino Básico: A concepção do tempo e a compreensão histórica das crianças e os contextos para o seu desenvolvimento [Universidade do Minho]. http://hdl.handle.net/1822/10153

VanSledright, B., \& Brophy, J. (1992). Storytelling, Imagination, and Fanciful Elaboration in Children's Historical Reconstructions. American Educational Research Journal, 29(4), 837859. http://doi.org/10.2307/1163409

Wallace, B. (2002). Teaching-Problem-solving and Thinking Skills in the Early Years: Working across the Curriculum. The earlier we start the better! In B. Wallace (ed.), Teaching Thinking Skills Across the Early Years. A practical approach for children aged 4-7 (pp. 1-42). London, UK: David Fulton Publishers.

Wood, L. y Holden, C. (2007). Enseyar història als més petits. Manresa, España: Zenobita. 